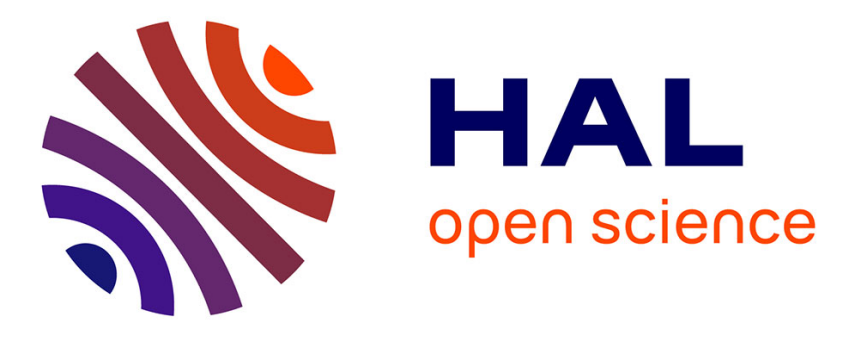

\title{
Investigation of LDPE converging flows using fieldwise measurements techniques
}

\author{
Ghalia Boukellal, D. Hertel, Rudy Valette, H. Münstedt, Jean-François
}

Agassant

\section{- To cite this version:}

Ghalia Boukellal, D. Hertel, Rudy Valette, H. Münstedt, Jean-François Agassant. Investigation of LDPE converging flows using fieldwise measurements techniques. 11th ESAFORM Conference on Material Forming, Apr 2008, Lyon, France. pp.Pages 687-690, 10.1007/s12289-008-0308-5 . hal00510260

\section{HAL Id: hal-00510260 \\ https: / hal-mines-paristech.archives-ouvertes.fr/hal-00510260}

Submitted on 18 Aug 2010

HAL is a multi-disciplinary open access archive for the deposit and dissemination of scientific research documents, whether they are published or not. The documents may come from teaching and research institutions in France or abroad, or from public or private research centers.
L'archive ouverte pluridisciplinaire HAL, est destinée au dépôt et à la diffusion de documents scientifiques de niveau recherche, publiés ou non, émanant des établissements d'enseignement et de recherche français ou étrangers, des laboratoires publics ou privés. 


\title{
Investigation of LDPE converging flows using fieldwise measurements techniques
}

\author{
G. Boukellal ${ }^{1}$, D. Hertel ${ }^{2}$, R. Valette ${ }^{1}$, H. Münstedt ${ }^{2}$, J.-F. Agassant ${ }^{1}$ \\ ${ }^{1}$ Ecole des Mines de Paris - ParisTech, Centre de Mise en forme des Matériaux - UMR CNRS 7635, \\ Rue Claude Daunesse, 06904 Sophia Antipolis, France \\ URL: www.cemef.ensmp.fr e-mail: rudy.valette@ensmp.fr \\ ${ }^{2}$ Lehrstuhl für Polymerwerkstoffe, Universität Erlangen-Nürnberg, \\ Martensstrasse 7, D-91058 Erlangen, Germany \\ URL: $\underline{w w w . l s p . u n i-e r l a n g e n . d e}$
}

\begin{abstract}
This study is devoted to the analysis of the secondary flow of a low-density polyethylene melt occurring in the entrance region of extrusion dies. From a processing point of view, the appearance of secondary flows can lead to flow instabilities (as observed for helical defect in circular channels) and polymer degradation (due to long residence times).

The flow is investigated in the entrance region of a flow channel by means of both Laser Doppler Velocimetry (LDV) and Flow Induced Birefringence (FIB) using a transparent slit die with a planar contraction of 14:1.

We focus on the influence of temperature $\mathrm{T}$ and flow rate $\mathrm{Q}$ on the vortex size of the secondary flow, which strongly depends on balance between shear and extensional rheological properties of the material, as previously observed by means of LDV.

The same dependence with $\mathrm{T}$ and $\mathrm{Q}$ is also observed on FIB patterns, in which one can identify a boundary line which exhibits a strong contrast in the birefringence pattern. This contrast could be related to the fact that molecular conformations (and consequently the birefringence pattern) change locally due to a different thermomechanical history, which indeed would occur close to the vortex boundary.
\end{abstract}

Key words: Low-density polyethylene, secondary flow, birefringence, laser-Doppler velocimetry.

\section{INTRODUCTION}

In this study, we investigate the secondary flow of a low density polyethylene (LDPE) in an abrupt contraction. This secondary flow, which is characterized by vortices in the entrance region of a die, is known to be due to strong differences between shear and extensional properties of the material. These differences are due to the high level of branching of the polymer molecules.

The global goal of this work is to combine both experimental and numerical studies in order to understand the influence of the rheological behavior on appearance of secondary flows for a specific grade of LDPE and then being able to identify the non-linear parameters of a suitable constitutive equation using complex flows. The first step of the study was to obtain precise measurements of kinematics and stresses, by using laser-Doppler velocimetry (LDV, at LSP) and flow induced birefringence (FIB, at CEMEF) techniques in transparent dies. These measurements have been carried out for different temperatures, which is known to have a strong influence on rheological behavior of the material.

\section{MATERIAL}

Material used is a commercial Lupolen $1840 \mathrm{H}$. Linear rheometry has been performed (Fig. 1) which shows that the terminal zone at low frequency can not clearly be identified. It would then be difficult to deduce the contribution of long chains to the rheological behaviour. Transient extensional viscosity curves (shown in Fig. 2) have been obtained using a Münstedt type rheometer. It clearly shows that the material exhibits strain hardening for very low values of the extensional rate, but no 


\section{EXPERIMENTAL TECHNIQUES}

Laser-Doppler velocimetry (LDV) is a measuring method which allows the absolute determination of the velocity distribution in flowing media. The functional principle is based on the Doppler effect. Two laser beams are focused within the flow channel and intersect each other in one spot. The result is a spatial interference pattern. When a small particle passes this pattern of alternating dark and bright planes, light is scattered back with a certain frequency corresponding to the light pattern and the velocity of the particle. The arising intensity pattern corresponds to the velocity component of the particle normal to the fringe pattern [1].

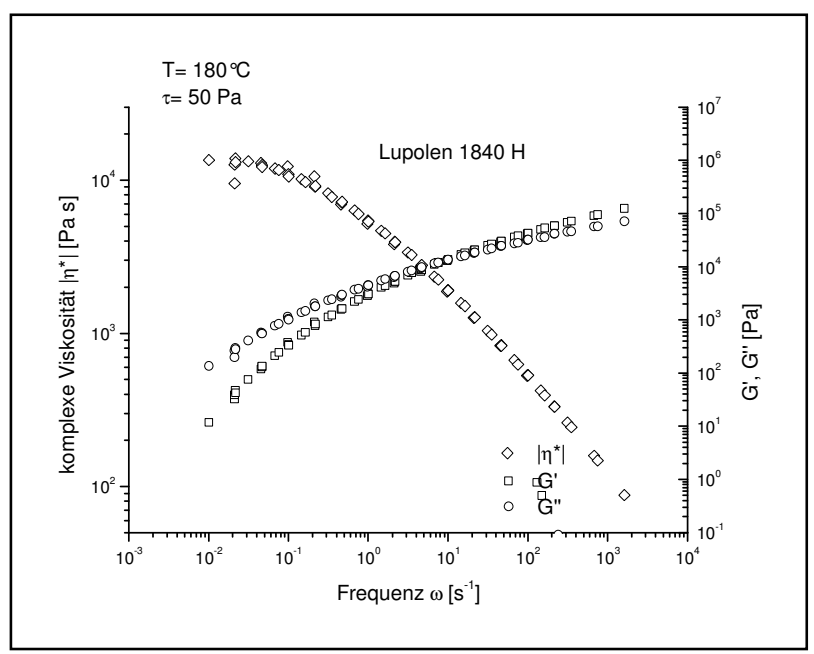

Fig. 1. Master curve at $180^{\circ} \mathrm{C}$ of the complex viscosity and modulus of the material.

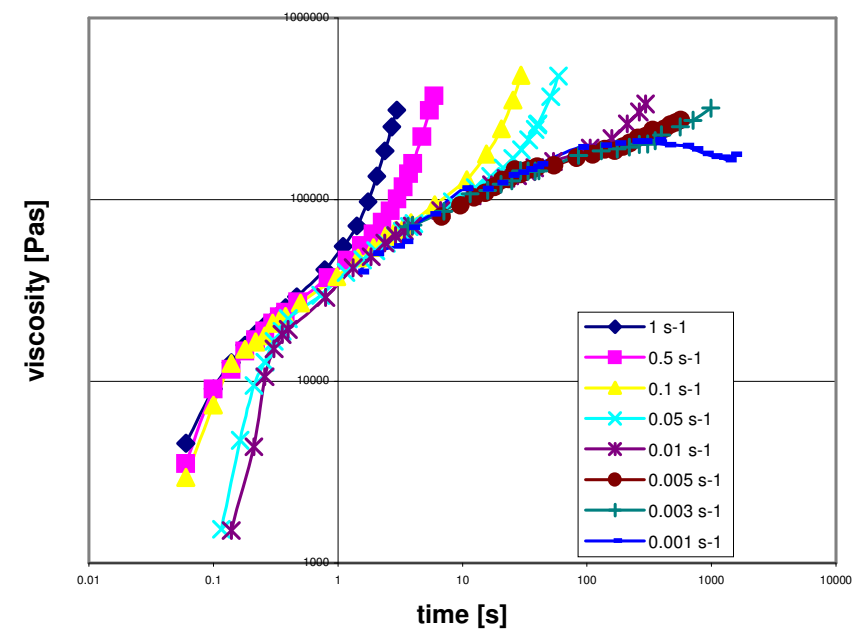

Fig. 2. Transient extensional viscosity for different strain rates at $180{ }^{\circ} \mathrm{C}$.
Laser-Doppler velocimetry (LDV) is a measuring method which allows the absolute determination of the velocity distribution in flowing media. The functional principle is based on the Doppler effect. Two laser beams are focused within the flow channel and intersect each other in one spot. The result is a spatial interference pattern. When a small particle passes this pattern of alternating dark and bright planes, light is scattered back with a certain frequency corresponding to the light pattern and the velocity of the particle. The arising intensity pattern corresponds to the velocity component of the particle normal to the fringe pattern [1].

The LDV-system enables the accurate determination of the velocity with high temporal $(\sim 5 \mu s)$ and spatial $(\sim 30 \mu \mathrm{m})$ resolution in flowing media. The velocity resolution is about $100 \mu \mathrm{m} / \mathrm{s}$, which allows a precise description of the velocities in the vortices.

At the LSP a special flow channel has been built up, which provides access to the flow in a slit die for high temperature and pressure ranges. The die consists of two inserts which can be exchanged so that the die geometry can be varied easily [2].

The experimental stress field is determined from isochromatic retardation bands of flow-induced birefringence. FIB is an optical characteristic exhibited by a molten polymer when it becomes anisotropic through the effect of orientation of the macromolecules within the flow field. In a plane flow, neglecting the wall effects, the level of anisotropy can be quantified according to the linear stress optical rule in term of principal stress difference (PSD). As such, the experimental PSD $(\Delta \sigma)$ can be computed from the FIB patterns by counting the relative retardation (or fringe order), $\mathrm{k}$, and assuming a typical value of the stress optical coefficient, $\mathrm{C}$, as follows:

$$
\Delta \sigma=\sigma_{I}-\sigma_{I I}=\frac{k \lambda}{C W}
$$

where $\lambda$ is the wavelength of the monochromatic light (in our experiments we used a sodium source with a wavelength $\lambda$ equals to $589 \mathrm{~nm}$ ), and $\mathrm{W}$ is the depth of the sample through which the light propagates [3].

In Fig. 3 the geometry of the flow cell and the used coordinate system are shown. The used contraction ratio, which is defined as

$C R=\frac{H_{\text {reservoir }}}{H_{\text {slit }}}$

is 12. All LDV and FIB measurements have been 
carried out in the center plane $(z=0)$ of the flow.

\section{RESULTS}

With the local velocity measurements of the LDV it is possible to characterize quantitatively the vortex. Schwetz et al. [4] showed measurements of the velocities within the vortices. These secondary flow regions are separated from the main flow region by a boundary line. They circulate with extremely low velocities compared to the main flow. An example of the secondary flow field measured in the $z=0$ plane, is shown in Fig. 4.

In the present work, the boundary line $y_{G}(x)$ was calculated by assuming that the mass balance from the walls to this line vanishes for any value of $x$ (the vortex being a closed region of the flow domain), and assuming that no flow rate in the $\mathrm{z}$ direction contributes to the mass balance.

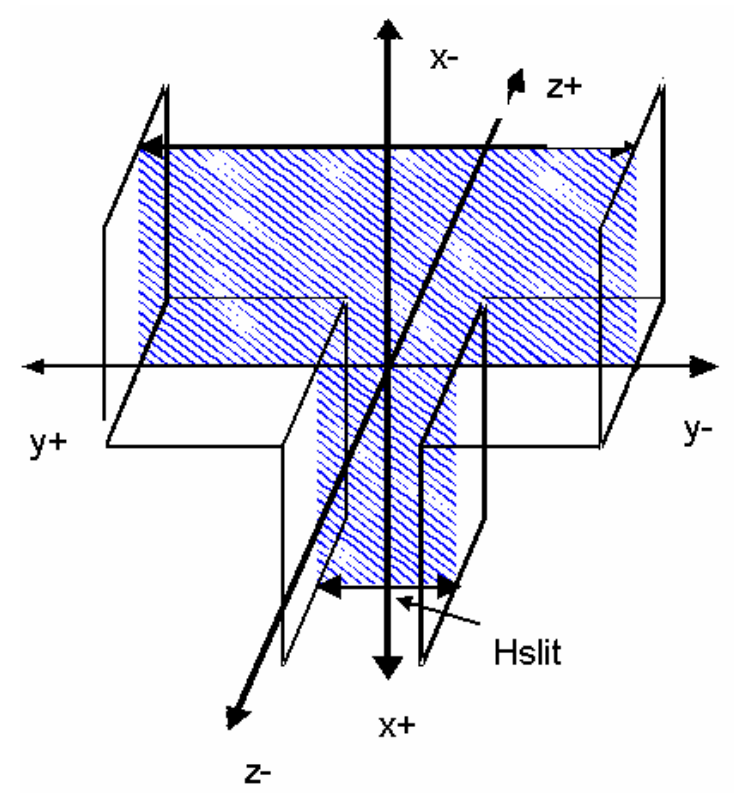

Fig. 3. Geometry of the flow cell and used coordinate system.

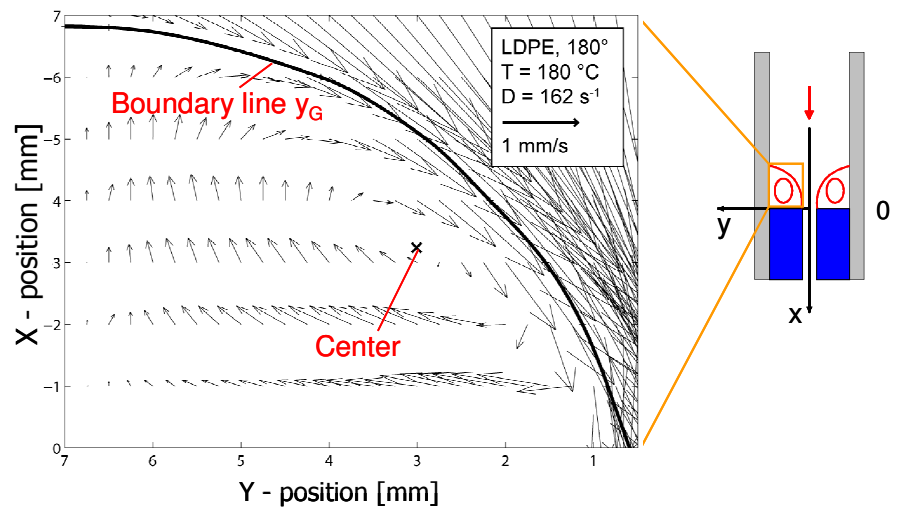

Fig. 4. Schematic of the quantification of the secondary flow (entrance vortex). formula:

$\int_{y=H_{R} / 2}^{y_{G}(x)} v_{x}(x, y) d y=q_{x}\left(y_{G}(x)\right)=0$,

which stands for all $x$. The boundary line is then given by:

$y_{G}(x)=q_{x}^{-1}(0)$.

The vortex center is the point, within the flow cell, where both $v_{x}$ and $v_{y}$ vanish within the flow cell. The vortex size $\mathrm{A}$ is defined as the area below the boundary line.

An interesting topic to investigate is the temperature-dependence of the vortex size. In figure 6 the vortex sizes for a constant mass throughput at different temperatures is shown. It is obvious that it runs through a maximum, which is at a temperature of about $190^{\circ} \mathrm{C}$.

It is noticeable a dependence with temperature is also observed on FIB patterns, in which one can identify a boundary line which exhibits a strong contrast in the pattern, as it is shown in figure 5.

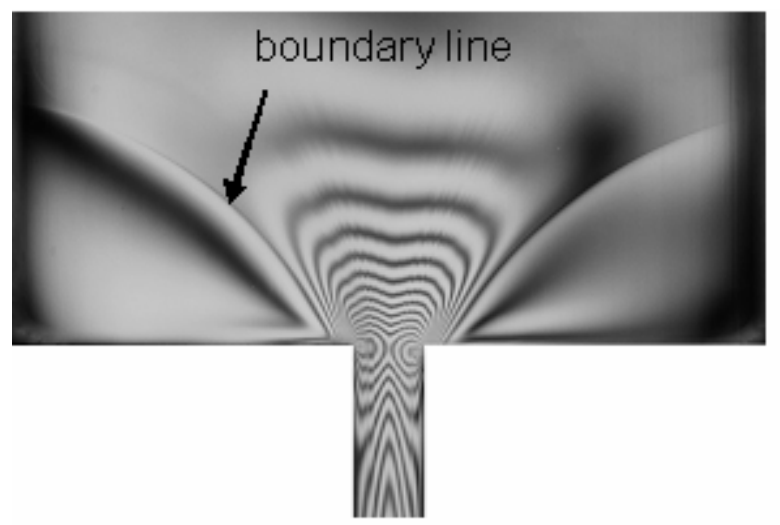

Fig. 5. Birefringence picture at $\mathrm{T}=180^{\circ} \mathrm{C}$ and $\mathrm{m}=0.3 \mathrm{~g} / \mathrm{s}$.

This contrast could come from the fact that molecular conformations (and consequently the birefringence pattern) changes locally due to a different thermomechanical history, which indeed would be the case through a vortex boundary. Assuming that the flow is not dependent on the $z$ direction, this line could then fit the vortex boundary.

Superposition of both LDV and FIB patterns show that the FIB boundary is systematically closer to the wall than the boundary line $y_{G}(x)$. It results that the surface below FIB boundary is slightly smaller than vortex sizes measured with LDV but varies in the same way with temperature. Following the same procedure as for LDV measurements, we have used this boundary line to calculate the area below it and compared it to LDV vortex sizes on figure 6. 


\section{DISCUSSION AND CONCLUSIONS}

The difference observed for the position of vortex boundaries for both patterns could be explained by the fact that 3D effects affect both measurements. Indeed, Sirakov et al. [5] have shown experimentally and numerically that flow kinematics exhibits strong variations in the $z$ direction in contraction flows of LDPE. In our case, these effects could affect both LDV measurements (in which the underlying assumption of $z$ invariance could affect mass balance calculations) and FIB measurements (as phase retardation is integrated all along the $z$ path).

In order to highlight these effects, both 3D LDV measurements and numerical simulations need to be carried out for the contraction flow. These studies, which are in progress, would allow to quantify the effect of rheological behavior on the appearance of vortices as this behavior is the key link between numerical and experimental investigations.

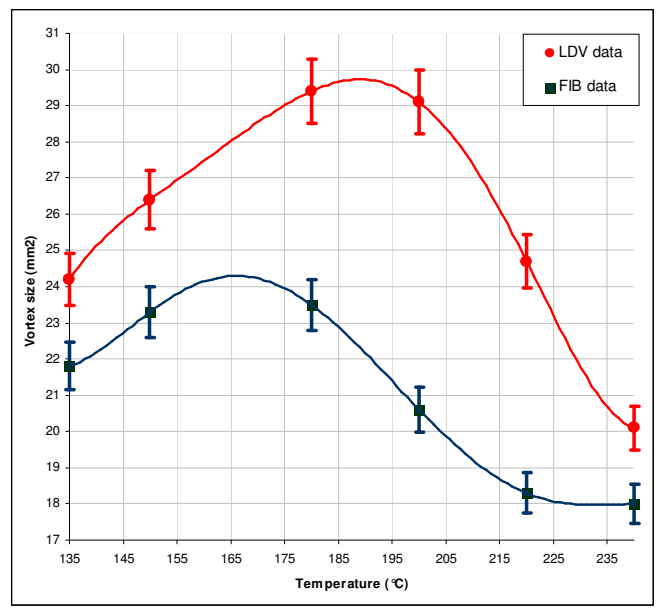

Fig. 6. Dependence of the vortex size on the temperature at $m=0.3 \mathrm{~g} / \mathrm{s}$, comparison between LDV and FIB data.

\section{REFERENCES}

1. Ruck B., Laser-Doppler-Anemometrie, AT-Fachverlag GmbH, Stuttgart, 1987.

2. Schmidt, M., E. Waßner, H. Münstedt, Setup and Test of a Laser-Doppler-Velocimeter for the Investigations of the Flow Behavior of Polymer Melts, Mech. Timedependent Mat. 3, 317-393 (1999).

3. Robert, L., B. Vergnes, Y. Demay, Flow birefringence study of the stick-slip instability during extrusion of high-density polyethylenes, J. Non-Newt. Fluid Mech. 112, 27-43, (2003).

4. Schwetz, M., H. Münstedt, M. Heindl, A. Merten, Investigations on the temperature dependence of the die entrance flow of various long-chain branched polyethylenes using laser-Doppler velocimetry, J. Rheol. 46 (4), 797-815 (2002).

5. Sirakov, I., A. Ainser, M. Haouche, J. Guillet, Threedimensional numerical simulation of viscoelastic contraction flows using the Pom-Pom differential constitutive model, J. Non-Newt. Fluid Mech. 126, 163173, (2005). 